\title{
Erratum to: Calculations of Freezing Point Depression, Boiling Point Elevation, Vapor Pressure and Enthalpies of Vaporization of Electrolyte Solutions by a Modified Three-Characteristic Parameter Correlation Model
}

\author{
Xinlei Ge $\cdot$ Xidong Wang
}

Published online: 17 April 2013

(C) Springer Science+Business Media New York 2013

Erratum to: J Solution Chem (2009) 38:1097-1117

DOI 10.1007/s10953-009-9433-0

Our attention has recently been drawn to a few typographical errors in the above paper.

On page 1099, Eq. 9, the temperature $T$ should not be in the left-hand side, and the negative sign in the square brackets in its right-hand-side should be a positive sign. Thus the correct equation is:

$$
R \ln a_{\text {liq }}=\Delta H_{0, T_{\mathrm{F}}}^{\text {fus }}\left(\frac{1}{T_{\mathrm{F}}}-\frac{1}{T_{\mathrm{F}}-\theta_{\mathrm{F}}}\right)+\Delta C_{p}^{\mathrm{fus}}\left[\ln \left(\frac{T_{\mathrm{F}}-\theta_{\mathrm{F}}}{T_{\mathrm{F}}}\right)+\frac{\theta_{\mathrm{F}}}{T_{\mathrm{F}}-\theta_{\mathrm{F}}}\right]
$$

On page 1099, Eq. 11, $T$ should not be in the last term of Eq. 11, and the correct equation is:

$$
\Delta H_{0, T_{F}}^{\text {fus }}\left(\frac{1}{T_{F}}-\frac{1}{T_{F}-\theta_{F}}\right)+\frac{\Delta C_{p}^{\text {fus }}}{2}\left(\frac{\theta_{F}}{T_{F}-\theta_{F}}\right)^{2}-R \ln a_{\text {liq }}=0
$$

On page 1099, Eq. 12, the gas constant $R$ was missing in the first term under the radical sign, and the correct equation is:

$$
\theta_{\mathrm{F}}=\frac{\Delta H_{0, T_{\mathrm{F}}}^{\mathrm{fus}}-2 R T_{\mathrm{F}} \ln a_{\mathrm{liq}}-\sqrt{2 \Delta C_{p}^{\mathrm{fus}} T_{\mathrm{F}}^{2} R \ln a_{\mathrm{liq}}+\left(\Delta H_{0, T_{\mathrm{F}}}^{\mathrm{fus}}\right)^{2}}}{2\left(\Delta H_{0, T_{\mathrm{F}}}^{\mathrm{fus}} / T_{\mathrm{F}}+0.5 \Delta C_{p}^{\mathrm{fus}}-R \ln a_{\mathrm{liq}}\right)}
$$

The online version of the original article can be found under doi:10.1007/s10953-009-9433-0.

X. Ge $(\bowtie)$

Department of Materials Science and Engineering, Royal Institute of Technology, 10044 Stockholm, Sweden

e-mail: xinleige@yahoo.com.cn

X. Wang

College of Engineering, Peking University, Beijing 100871, China 
On page 1100, Eq. 16, $T$ should not be in the left-hand-side and negative sign was missing in the first term of right-hand-side, and the correct equation is:

$$
-R \ln a_{\mathrm{liq}}=-\Delta H_{0, T_{\mathrm{B}}}^{\mathrm{vap}}\left(\frac{1}{T_{\mathrm{B}}}-\frac{1}{T_{\mathrm{B}}+\theta_{\mathrm{B}}}\right)+\Delta C_{p}^{\mathrm{vap}}\left[\ln \left(\frac{T_{\mathrm{B}}+\theta_{\mathrm{B}}}{T_{\mathrm{B}}}\right)-\frac{\theta_{\mathrm{B}}}{T_{\mathrm{B}}+\theta_{\mathrm{B}}}\right]
$$

On page 1100, Eq. 17, $R$ is missing in the second term under the radical sign, and the correct equation is:

$$
\theta_{\mathrm{B}}=\frac{-\Delta H_{0, T_{\mathrm{B}}}^{\mathrm{vap}}-2 R T_{\mathrm{B}} \ln a_{\mathrm{liq}}+\sqrt{\left(\Delta H_{0, T_{\mathrm{B}}}^{\mathrm{vap}}\right)^{2}-2 \Delta C_{p}^{\mathrm{vap}} T_{\mathrm{B}}^{2} R \ln a_{\mathrm{liq}}}}{2\left(\Delta H_{0, T_{\mathrm{B}}}^{\mathrm{vap}} / T_{\mathrm{B}}+0.5 \Delta C_{p}^{\mathrm{vap}}-R \ln a_{\mathrm{liq}}\right)}
$$

In all instances, the subsequent calculations in the paper used the correct equations as listed above and not the original equations with errors. Thus the results presented there are unchanged. 